\title{
REFRACTION ANGLE CALCULATION IN MULTILAYERED ICE FOR WIDE-BEAM AIRBORNE RADAR
}

\author{
Alvaro Arenas-Pingarron ${ }^{1}$, Paul V. Brennan ${ }^{1}$, Hugh Corr $^{2}$ \\ ${ }^{1}$ Department of Electronic \& Electrical Engineering, University College London, London WC1E 7 JE, UK \\ ${ }^{2}$ British Antarctic Survey, High Cross, Madingley Road, Cambridge CB3 0ET, UK
}

\begin{abstract}
We propose an algorithm to calculate the refraction path travelled by a signal from a source to a remote scatterer, through a horizontally parallel multilayer medium with vertical-varying refractive index. The exact solution in a simple two-layered medium requires solving a degree-4 polynomial according to Snell's law, meaning a timedemanding solution. For narrow-beam sources a small-angle approximation is commonly used. In this new technique, we transformed the governing equations until the system can be solved by intersection of two curves. As these are monotonic with opposite slope, the crossing point can be estimated by iteration, with a minimum search algorithm. This method has been applied to focus synthetic aperture radar images of the Antarctic bedrock, improving the detected response in the wide-beam case, and reducing time regarding to compute the polynomial solution.
\end{abstract}

Index Terms - Back-projection, radar, refraction, SAR, Snell's law.

\section{INTRODUCTION}

A key parameter in signal processing of antenna arrays is the estimation of the path followed by the signals. The synthetic aperture radar (SAR) is a particular case, where the relative phase of the channels in the array depend on the radar trajectory and the target location, and will be used for calculating either the range-azimuth reference, the motion compensation or the cross-track beamforming functions.

The incidence angle of an electromagnetic (EM) wave through a multilayer medium is obtained through the equations defined by Snell's law and the ground distance between the source and the target. For the simplest case of radar and target each in a different uniform medium, the ground range distance from an airborne SAR to the refraction point on the air-ice interface corresponds with the roots of a degree-4 polynomial [1], and from this distance, the elevation angle results. However, solving these roots (together with discarding solutions) is computationally timedemanding, and even more so when taking into account 3Ddistributed targets. For SAR image processing, a smallangle approximation has been used [2-3], faster than the iterative method, but less accurate for large angles. For example, when a wide aperture is needed for high azimuth resolution, or a large angle of incidence is used for squinted processing, useful to detect the steep internal ice layers. Other approaches have been applied, such as using a lookup table [4], from which the elevation angle or ground range distance can be measured after interpolation. However, if parameters different from those for producing the table are needed, the values must be recalculated, and thus inflexible.

In ice-sounding applications, the convenience of at least two ice layers was put forward in [5], adding complexity in airborne techniques.

\section{MODEL DESCRIPTION}

The speed of EM wave propagation varies inversely with refractive index and density of the medium. In ice, the wave propagation speed decreases with depth, because the air concentration reduces (more present in the precipitating snowflakes) and the ice becomes more compacted due to higher pressure. In the shallowest layer, known as firn and which might extend down to about $150 \mathrm{~m}$ depth, the propagation speed is higher, decreasing until a solid ice region, known as glacier ice, is reached, with an expected constant refractive index of about 1.78 [6], compared to a minimum value of about 1.20 for ice on surface [5].

For simplicity, the ice medium is usually treated as a single uniform layer, adding a correction of $10 \mathrm{~m}$ for depth estimation, to account for the faster speed of propagation through firn [6]. Since refractive index increases with depth in the firm, a more realistic model should include different ice layers, as depicted in Fig. 1, where $H$ is the radar height over the surface, $R_{G}$ and $R_{G P}$ are, respectively, the ground distances from radar to target and refraction point on surface, $\theta_{0}$ the elevation angle in the source medium (air, for airborne SAR) and $d_{i}, n_{i}, \theta_{i}$ and $R_{G i}$ are, respectively, the thickness, the relative refractive index regarding the source medium, the incidence angle and the ground distance covered, of the $i$-th layer, out of $L$, in which ice is divided.

The refraction path and elevation angle is calculated from the distance $R_{G P}$, as

$$
\theta_{0}=\tan ^{-1}\left(R_{G P} / H\right)
$$




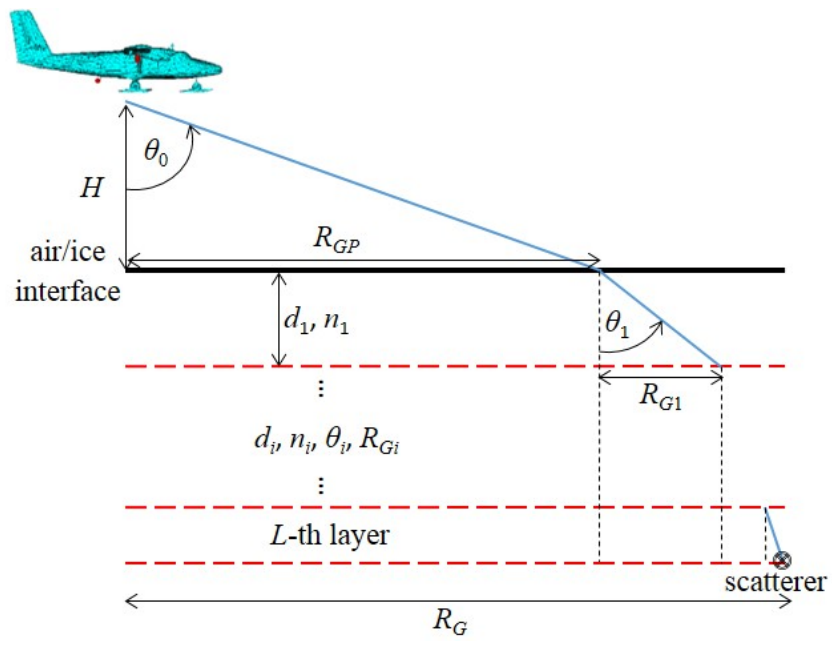

Fig. 1. Refraction path geometry in a multilayer ice medium.

\section{PREVIOUS METHODS}

Snell's law establishes that a wave propagating through different media follows the fastest path. The solution is found by equating to zero the derivative (regarding the desired parameter) of the one-trip delay, which will be the polynomial solution. An approximation of Snell's law leads to small-angle calculations.

\subsection{Exact polynomial solutions}

Snell's law relates each layer $i$ to the first medium (air) by

$$
\sin \left(\theta_{0}\right)=n_{i} \cdot \sin \left(\theta_{i}\right), 1 \leq i \leq L
$$

On the other hand, for covering the ground distance $\mathrm{R}_{\mathrm{G}}$,

$$
R_{G}=R_{G P}+\sum_{i=1}^{L} R_{G i} .
$$

The case for the single-layer ice medium $(L=1)$ was solved in [1] as a degree-4 polynomial, which, with the notation of Fig. 1, can be expressed in a compact form as

$$
R_{G 1}^{2}=\frac{d_{1}^{2} R_{G P}^{2}}{P_{1}}
$$

with $P_{1}$ the degree-2 polynomial on $R_{G P}$

$$
P_{1}\left(R_{G P}\right)=\left(n_{1}^{2}-1\right) R_{G P}^{2}+n_{1}^{2} H^{2}
$$

Because the layers are commutative when they are parallel, for each of the $L$ layers exist analogous equations to (4-5). If in (3) the term $R_{G 1}$ within the summation is cleared, and $R_{G i}$ for every layer is expressed in terms of $R_{G \mathrm{P}}$ with (4), it leads to the equation for the general case of $L$ ice layers

$$
\frac{d_{1}^{2} R_{G P}^{2}}{P_{1}}=\left(R_{G}-R_{G P}-R_{G P} \cdot \sum_{i=2}^{L} \frac{d_{i}}{\sqrt{P_{i}}}\right)^{2} .
$$

To obtain a polynomial of positive integer powers, (6) must be rearranged until the $P_{i}$ terms appear in the numerator, yielding an equation of maximum positive integer degree $2 \cdot(L+1)$, although still with rational powers. As in (6) there are $L-1$ square roots, also $L-1$ squaring operations are required to transform the previous polynomial of $2 \cdot(L+1)$ degree, until being all powers positive integers. Because each square doubles the polynomial degree, the total factor is $2^{L-1}$. All together makes a polynomial of degree

$$
N=2(L+1) \cdot 2^{L-1}=(L+1) \cdot 2^{L} .
$$

This technique gives an exact solution in the form of a polynomial, although it is computationally demanding, since generally it requires finding the eigenvalues of a matrix. $L=0$ corresponds to the trivial solution $R_{G P}=R_{G}$, with degree 1 . Considering firn and glacier layer $(L=2)$, the degree is 12 , which could be a problem not only in real time applications, but also in off-line processing when the signal paths must be calculated often, making interesting to approximate the exact calculations. The compact version for $L=2$ is

$$
\begin{gathered}
4 R_{G P}^{2}\left(R_{G}-R_{G P}\right)^{2} d_{2}^{2} P_{1}^{2} P_{2} \\
=\left(\left(d_{1}^{2} P_{2}-d_{2}^{2} P_{1}-P_{1} P_{2}\right) R_{G P}^{2}+2 R_{G} P_{1} P_{2} R_{G P}-R_{G}^{2} P_{1} P_{2}\right)^{2} .
\end{gathered}
$$

\subsection{Small-angle approximation}

An approximation for small angles can be applied [2], in which the sine of the incidence angles in free space and ice is approximated by the tangent. Rewriting (3) as

$$
H \cdot \tan \left(\theta_{0}\right)+\sum_{i=1}^{L} d_{i} \cdot \tan \left(\theta_{i}\right)=R_{G}
$$

and approximating (2) by means of the tangent

$$
R_{G P}=H \cdot \tan \left(\theta_{0}\right) \approx R_{G} \cdot\left(1+\frac{1}{H} \cdot \sum_{i=1}^{L} \frac{d_{i}}{n_{i}}\right)^{-1} .
$$

Although this solution offers a fast analytical way, it has two main drawbacks. Firstly, it might be not valid for squinted processing, where the main Doppler beam illuminates an area far from nadir, pointing forwards or backwards. Secondly, the validity of this approximation is wavelength dependent, as the calculated range to the target will have a corresponding phase shift error. Besides, with relatively low flying aircrafts, the approximation is not valid for shallow targets. 


\section{ITERATIVE ESTIMATION}

The new approach consists of finding the intersection point of unidimensional functions, with the same bounded definition domain. Since this point is unique, an optimized algorithm can be used. The advantage of this technique is that it works for all angles, with an accuracy according to the number of iterations performed.

\subsection{Derivation}

With new variables $x, y_{i}, a_{i}$ and $b_{i}$ defined as

$$
\begin{gathered}
x=\frac{H}{R_{G}} \tan \left(\theta_{0}\right), \quad y_{i}=\frac{d_{i}}{R_{G}} \tan \left(\theta_{i}\right), \\
a_{i}=\frac{d_{i}}{R_{G} \sqrt{\left(n_{i}^{2}-1\right)}}, \quad b_{i}=\frac{n_{i} \cdot H}{R_{G} \sqrt{\left(n_{i}^{2}-1\right)}}
\end{gathered}
$$

being $x$ and $y_{i}$ within the interval $[0,1]$, readily checked with (9). The latter is transformed to

$$
x+\sum_{i=1}^{L} y_{i}=1
$$

and the set of $L$ equations in (2) to

$$
y_{i}=\frac{a_{i} x}{\sqrt{b_{i}^{2}+x^{2}}}, \quad 1 \leq i \leq L .
$$

Clearing $y_{1}$ in (12) and with (13), it results

$$
\begin{aligned}
y_{1} & =f_{1}(x)=1-x \cdot\left(1+\sum_{i=2}^{L} \frac{a_{i}}{\sqrt{b_{i}^{2}+x^{2}}}\right) \\
& =f_{2}(x)=\frac{a_{1} x}{\sqrt{b_{1}^{2}+x^{2}}} .
\end{aligned}
$$

The solution to the system is the crossing point $x_{c}$

$$
\begin{aligned}
x_{c} & =\left\{x \in[0,1] \mid g(x)=f_{1}(x)-f_{2}(x)=0\right\} \\
& =\left\{x \in[0,1]|| g(x)|=| f_{1}(x)-f_{2}(x) \mid=0\right\}
\end{aligned}
$$

to finally obtain the ground distance from radar to surface as

$$
R_{G P}=H \cdot \tan \left(\theta_{0}\right)=x_{c} \cdot R_{G} .
$$

\subsection{Solution search algorithms}

Inspection of $f_{1}(x)$ and $f_{2}(x)$ in (14) allows us to determinate a method to calculate $x_{c}$ : since $f_{1}(x)$ is strictly monotonically increasing and $f_{2}(x)$ decreasing, the solution $g(x)=0$ is unique. If the absolute value of $g(x)$ is considered, the solution will be the minimum of $|g(x)|$, also unique. This makes possible to apply an optimized algorithm to find the solution by means of $g(x)$, with zero-crossing, or $|g(x)|$ with the minimum. In Fig. 2 are plotted these functions, for the case of $L=2$, with a firn layer of $d_{1}=150 \mathrm{~m}$ and $n_{1}=1.5$, when pointing a target $2 \mathrm{~km}$ below the firn, with a radar at a ground distance of $300 \mathrm{~m}$ and height of $500 \mathrm{~m}$. Those points where $y_{1}$ is not between the interval $[0,1]$ are not valid (it would mean the EM wave refracts in the opposite direction, only possible in case the layers are not parallel), so the interval where to find $x_{c}$ can be reduced.

The case for $L=1$ makes $f_{1}(x)=1-x$, a linear function independent of the ice layer. Besides, for $b_{1} \gg 1$, also $f_{2}(x)$ can be regarded as linear, and then $x_{c} \approx 1 /\left(1+a_{1} / b_{1}\right)$.

The 'Bisection' method [7], based on the divide-andconquer technique, was used to calculate (15), comparing different probe points of the function to iteratively approach the zero-crossing or minimum point, reducing the search interval at each step.

Within an interval, the probability density of finding the minimum is assumed to be uniform. This, together with the nature of $g(x)$ (strictly monotonically decreasing) and $|g(x)|$ (increasing derivative), allows the rejection of a region after evaluating a probe point, located in the interval middlepoint. With the 'Bisection' method, the ratio between the lengths of the current search interval and the next one is optimized with a value of 2 (uniform probability). The difference when using (15a) and (15b) is that the former means a single evaluation of $g(x)$, while the latter needs the derivative of $|g(x)|$, which might be a difficult expression if a single point is wanted with the analytical form, or require to differentiate two points of $|g(x)|$. It is also important to consider the complexity of the evaluation of $g(x)$ and $|g(x)|$, which efficiency depends on the software used. Other option is to work with equivalent expressions: $g(x)$ is the difference of $f_{1}(x)$ and $f_{2}(x)$, but the latter contains a root square, and then it might be more efficient to square $f_{1}(x)$ and $f_{2}(x)$.

The number of iterations determines the resolution in the abscise interval $[0,1]$ : as the ratio of the interval length after consecutive iterations is 2, after $K$ steps the ratio is $2^{K+1}$ (with uniform probability density, for $K=0$ the point 0.5 would be the solution), and thus an error of $2^{-(K+1)}$ occurs.

\section{RESULTS AND COMPARISONS}

The order of complexity of the polynomial solutions with degree $N$, is $O\left(N^{2}\right)$, and expressing $N$ in terms of the number of layers $L$, according to (7) results in $O\left(L^{2} 2^{2 L}\right)$. The smallangle approximation has an order $O(L)$, since each layer adds a summand in (10). In the intersection approach, a new layer also adds a summand to $f_{1}(x)$ in (14), and with $K$ iterations, the complexity is $O(K \cdot L)$.

Fig. 3 compares the estimated incidence angle of the airice interface $\theta_{0}$, for the small-angle and intersection techniques, subtracting to their value the polynomial solution, the latter expected to be exact. The model 


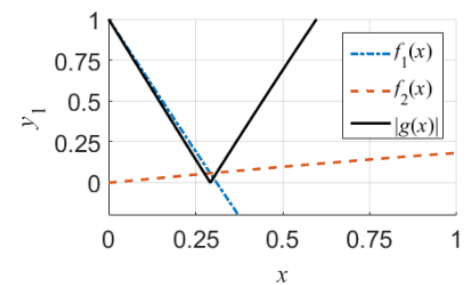

Fig. 2. Intersection point and curves for $H=500 \mathrm{~m}, R_{G}=300 \mathrm{~m}$, $n_{1}=1.5, n_{2}=1.78, d_{1}=150 \mathrm{~m}$ and $d_{2}=2 \mathrm{~km}$.

parameters are the same as for Fig. 2, except for the ground distance $R_{G}$, now from 0 to $500 \mathrm{~m}$. The small-angle method has an error increasing with the ground distance, while the intersection approach, plotted with 10 and 15 iterations, presents an oscillating deviation.

Fig. 4 shows the range-Doppler SAR response of a region with approximately flat bedrock, at $3.4 \mathrm{~km}$ depth, in data taken over Recovery Glacier, using the British Antarctic Survey PASIN (Polarimetric Airborne Scientific Instrument) radar at $150 \mathrm{MHz}$, with height above surface $340 \mathrm{~m}$, speed $55.2 \mathrm{~m} / \mathrm{s}$, pulse repetition frequency after presuming $78.125 \mathrm{~Hz}$ and sampling frequency $24 \mathrm{MHz}$. Overlaid on the image are the range-Doppler expected responses after estimating the path with the small-angle approximation (dashed) and intersection method (dot and dashed) assuming a uniform ice-thickness with 1.78 the refractive index, while the solid line represents the case with a firn of $d_{1}=100 \mathrm{~m}$ and $n_{1}=1.3$.

\section{CONCLUSION}

The proposed approach allows a fast estimation of signal paths when travelling through a stratified medium made up of parallel layers, applying any of the well-known minimum search algorithms. Besides, its efficiency can be improved within each iteration, processing each layer with parallel computing. Although this work is framed in ice-sounding, it can be applied also to other fields, such as soil-sounding for mine and water detection or atmospheric radars. The next step will be the development of a similar algorithm to take into account non-parallel layers.

\section{ACKNOWLEDGMENT}

We thank C.N. Robinson and R.J. Bullock for the collection and processing of raw data, and L.B. Lok and K. Nicholls for the suggestions and reviews.

\section{REFERENCES}

[1] F. Hélière, C.-C. Lin, H. Corr and D. Vaughan, "Radio Echo Sounding of Pine Island Glacier, West Antarctica: Aperture Synthesis Processing and Analysis of Feasibility From Space", Geoscience and Remote Sensing, IEEE Transactions on, 45(8), pp. 2573-2582, Aug. 2007.

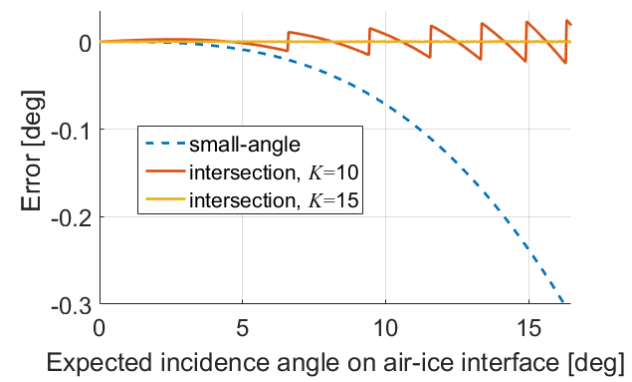

Fig. 3. Deviation of the estimated refraction angle on air-ice interface, for small-angle approximation (dashed) and intersection method (solid) with 10 and 15 steps, with the same parameters as in Fig. 2, but with $R_{G}$ from 0 to $500 \mathrm{~m}$.

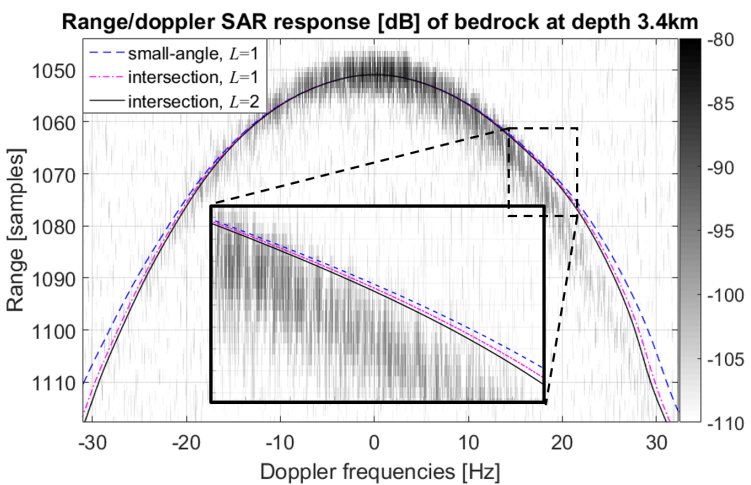

Fig. 4. Range-doppler SAR response $[\mathrm{dB}]$ of bedrock, matching with path estimation: 1 layer, with small-angle approximation (dash) and intersection method (dot-dash); and 2 layers (solid), with an intermediate firn layer of $100 \mathrm{~m}$ and refractive index 1.3 .

[2] A. Kusk, and J. Dall, "SAR focusing of P-band ice sounding data using back-projection," 2010 IEEE International Geoscience and Remote Sensing Symposium, Honolulu, HI, pp. 4071-4074, 2010.

[3] J. J. Legarsky, S. P. Gogineni, and T. L. Akins, "Focused Synthetic Aperture Radar Processing of IceSounder Data Collected Over the Greenland Ice Sheet", IEEE Transactions on Geoscience and Remote Sensing, 39(10), pp. 2109-2117, Oct. 2001.

[4] T. Newman, "Application of Synthetic Aperture Techniques to Radar Echo Soundings of the Pine Island Glacier, Antarctica", Ph.D. dissertation, Dept. Earth Sci., University College London, UK, 2011.

[5] L.A. Rasmussen, "Refraction Correction for Radio Echo-Sounding of Ice Overlain by Firn", Journal of Glaciology, vol.32, n0. 111, pp. 193-194, 1986.

[6] J. A. Dowdeswell, and S. Evans, "Investigations of the form and flow of ice sheets and glaciers using radio-echo sounding", Reports on Progress in Physics, vol. 67, pp. 1821-1861, 2004.

[7] Burden, Richard L., Faires, J. Douglas, "Solutions of Equations in One variable", Numerical Analysis (8th ed.), Thomson Brooks/Cole, 2005. 